\title{
FURTHER EXPLORATION OF RIEMANN'S FUNCTIONAL EQUATION
}

\author{
MichaEL MiLgRAM
}

\begin{abstract}
A previous exploration of the Riemann functional equation that focussed on the critical line, is extended over the complex plane. Significant results include a simpler derivation of the fundamental equation obtained previously, and its generalization from the critical line to the complex plane. A simpler statement of the relationship that exists between the real and imaginary components of $\zeta(s)$ and $\zeta^{\prime}(s)$ on opposing sides of the critical line is developed, reducing to a simpler statement of the same result on the critical line. An analytic expression is obtained for the sum of the arguments of $\zeta(s)$ on symmetrically opposite sides of the critical line, reducing to the analytic expression for $\arg (\zeta(1 / 2+i \rho))$ first obtained in the previous work. Relationships are obtained between various combinations of $|\zeta(s)|$ and $\left|\zeta^{\prime}(s)\right|$, particularly on the critical line, and it is demonstrated that the difference function $\arg (\zeta(1 / 2+i \rho))-\arg \left(\zeta^{\prime}(1 / 2+i \rho)\right)$ uniquely defines $|\zeta(1 / 2+i \rho)|$. A comment is made about the utility of such results as they might apply to putative proofs of Riemann's Hypothesis (RH).
\end{abstract}

Mathematics subject classification (2010): 11M06, 11M26, 11M99, 26A09, 30B40, 30E20, 30C15, 33C47, 33B99, 33F99.

Keywords and phrases: Zeta function, Riemann Hypothesis, Riemann's functional equation, argument of Zeta function, argument of derivative of Zeta function, zeros of Zeta function.

\section{REFERENCES}

[1] R. P. BREnT, On asymptotic approximations to the log-Gamma and Riemann-Siegel theta functions, ArXiv e-prints, Sept. (2016), https://arxiv.org/abs/1609.03682v2.

[2] Maplesoft, a division of Waterloo Maple Inc., Maple.

[3] M. S. Milgram, Notes on the zeros of Riemann's Zeta function, 2011, http://arxiv.org/ abs/0911.1332.

[4] M. S. Milgram, Integral and series representations of Riemann's Zeta function, Dirichlet's Eta function and a medley of related results, Journal of Mathematics, Article ID 181724, 2013, http://dx.doi.org/10.1155/2013/181724.

[5] Michael Milgram, Exploring Riemann's functional equation, Cogent Mathematics, 3 (1): 1179246, 2016, http://dx.doi.org/10.1080/23311835.2016.1179246.

[6] S. NaZARdonyavi And S. Yakubovich, On an inequality for the Riemann Zeta-function in the critical strip, ArXiv e-prints, June (2012), https ://arxiv.org/abs/1206.1801.

[7] NIST Digital Library of Mathematical Functions, http://dlmf .nist.gov/, Release 1.0.9 of 201408-29.

[8] F. W. J. Olver, D. W. Lozier, R. F. Boisvert, and C. W. Clark, editors, NIST Handbook of Mathematical Functions, Cambridge University Press, New York, NY, 2010, Print companion to [7].

[9] SAidak F. AND P. Zvengrows Ki, On the modulus of the Riemann zeta function in the critical strip, Math. Slovaca, 53: 145-172, 2003.

[10] Robert SPIRA, An inequality for the Riemann Zeta function, Duke Math. J., 32 (2): 247-250, 06 1965.

[11] Robert SPIRA, Zeros of $\zeta^{\prime}(s)$ and the Riemann Hypothesis, Illinois J. Math., 17 (1): 147-152, 03 1973.

[12] G. K. SRinivasan And P. Zvengrowski, On the horizontal monotonicity of $|\Gamma(s)|$, Canad. Math. Bull, 54 (3): 538-543, 2011. 
[13] E. C. Titchmarsh and D. R. Heath-Brown, The Theory of the Riemann Zeta-Function, Oxford Science Publications, Oxford, Second edition, 1986. 